Conclusion: Combining a diverse analysis of BAL proteins with the rich dataset available from SSc-ILD patients participating in SLS I, the study findings suggest the involvement of distinct biologic pathways, inter-related networks, and specific biologic signatures associated with unique radiographic features of ILD. The relationship of these factors to other SSc disease features, patient outcomes and as predictors of treatment responses will be studied in future analyses. References:

[1] Tashkin DP, et al. NEJM 2006.

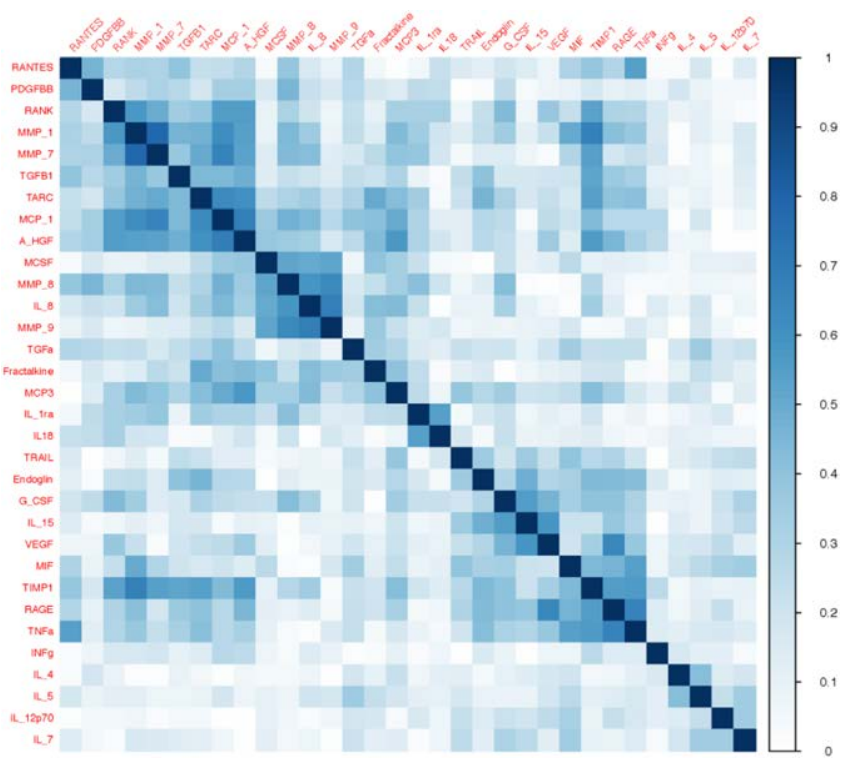

Figure. Correlation heatmap of BAL proteins associated with at least 2 clinical measures of ILD in SSc patients. Absolute correlations are depicted, and darker colors signify stronger correlations.

Disclosure of Interests: Elizabeth Volkmann Grant/research support from: Forbius, Corbus Pharmaceuticals, Consultant of: Boehringer Ingelheim, Forbius, Speakers bureau: Boehringer Ingelheim, Donald Tashkin: None declared, Ning Li: None declared, Grace Kim: None declared, Jonathan Goldin: None declared, Airi Harui: None declared, Michael Roth Grant/research support from: Genentech/ Roche

DOI: 10.1136/annrheumdis-2020-eular.2526

\section{SAT0287 \\ AMPLIFICATION OF THE PRO-FIBROTIC JAK2-STAT3 SIGNALING AXIS BY TGFB-INDUCED EPIGENETIC SILENCING OF SOCS3}

C. Dees ${ }^{1}$, S. Poetter ${ }^{1}$, Y. Zhang ${ }^{1}$, C. Bergmann ${ }^{1}$, X. Zhou ${ }^{1}$, M. Luber ${ }^{1}$, E. Karouzakis ${ }^{2}$, A. Ramming ${ }^{1}$, O. Distler ${ }^{2}$, G. Schett ${ }^{1}$, J. Distler ${ }^{1}{ }^{1}{ }^{1}$ FriedrichAlexander-University (FAU) Erlangen-Nürnberg, Department of Internal Medicine 3 - Rheumatology and Immunology, Universitätsklinikum Erlangen, Erlangen, Germany; ${ }^{2}$ Department of Rheumatology, Center of Experimental Rheumatology, University Hospital of Zurich, Zurich, Switzerland

Background: Tissue fibrosis caused by a pathological activation of fibroblasts is a major hallmark of systemic sclerosis (SSc). Epigenetic gene silencing of anti-fibrotic genes is thought to play a central role to establish the persistently activated phenotype of fibroblasts independent of external stimuli such as TGF $\beta$, which has been identified as key-mediator of fibroblast activation.

Objectives: The aims of the present study were to investigate whether the aberrant activation of JAK2-STAT3 signaling in fibrosis might be caused by epigenetic silencing of SOCS expression and whether re-establishment of the endogenous, SOCS-dependent control of JAK / STAT signaling may prevent aberrant fibroblast activation and ameliorate tissue fibrosis.

Methods: The methylation status of SOCS3 in fibroblasts was evaluated by methylation-specific PCR and MeDIP assays. 5-aza-2-deoxycytidine (5-aza) and siRNA was used to inhibit DNA methyltransferases (DNMTs) in vitro and in vivo. Knockdown and overexpression experiments served to analyze the mechanism of action in cultured fibroblasts. Fibroblast-specific knockout mice were additionally used to analyze the role of SOCS3 and DNMTs in vivo.

Results: Chronically increased levels of TGF $\beta$ reduced the expression of SOCS3 in normal fibroblasts to a level also found in SSc fibroblasts. Consistently, the expression of SOCS3 was severely downregulated in skin of SSc patients compared to healthy individuals with only minor differences between limited and diffuse cutaneous SSc. Methylation analyses demonstrated a prominent promoter hypermethylation of SOCS3 in SSc fibroblasts and in normal fibroblasts exposed to persistently high levels of TGF $\beta$. Increased DNMT activity and a time-dependent induction of DNMT3A and DNMT1 expression upon chronic exposure to TGF $\beta$ resulted in promoter hypermethylation of SOCS3. Knockdown of SOCS3 induced an SSc-like phenotype in normal dermal fibroblasts with increased activation of JAK2-STAT3 signaling, enhanced expression of myofibroblast markers, increased collagen release, and aggravated experimental tissue fibrosis with increased activation of JAK2-STAT3 signaling. This effect was mimicked by overexpression of mutant JAK2 with mutations in the SOCS3 binding motif. Vice versa, forced overexpression of SOCS3 reduced TGF $\beta$-mediated fibroblast activation and ameliorated the endogenous activation of SSc fibroblasts. Pharmacological inhibition or selective knockdown of DNMTs restored the normal expression of SOCS3, reduced fibroblast activation and collagen release, blocked STAT3-responsive transcription, and exerted potent antifibrotic effects in bleomycin- and TBRI ${ }^{\text {act }}$-induced dermal fibrosis. In addition, treatment with 5-aza or knockdown of either DNMT1 or DNMT3A induced regression of established fibrosis.

Conclusion: These findings identify a novel pathway of epigenetic imprinting of fibroblasts in fibrotic disease with translational implications for the development of new targeted therapies in fibrotic diseases. We demonstrate that the chronic activation of TGF $\beta$ signaling in fibrotic diseases perturbs the epigenetic control of STAT signaling by DNMT-induced silencing of SOCS3 expression. Our data migh thus strengthen the scientific rational for targeting DNA methylation in fibrotic diseases.

Disclosure of Interests: Clara Dees: None declared, Sebastian Poetter: None declared, Yun Zhang: None declared, Christina Bergmann: None declared, xiang zhou: None declared, Markus Luber: None declared, Emmanuel Karouzakis: None declared, Andreas Ramming Grant/research support from: Pfizer, Novartis, Consultant of: Boehringer Ingelheim, Novartis, Gilead, Pfizer, Speakers bureau: Boehringer Ingelheim, Roche, Janssen, Oliver Distler Grant/research support from: Grants/Research support from Actelion, Bayer Boehringer Ingelheim, Competitive Drug Development International Ltd. and Mitsubishi Tanabe; he also holds the issued Patent on mir-29 for the treatment of systemic sclerosis (US8247389, EP2331143)., Consultant of: Consultancy fees from Actelion, Acceleron Pharma, AnaMar, Bayer, Baecon Discovery Blade Therapeutics, Boehringer, CSL Behring, Catenion, ChemomAb, Curzion Pharmaceuticals, Ergonex, Galapagos NV, GSK, Glenmark Pharmaceuticals Inventiva, Italfarmaco, iQvia, medac, Medscape, Mitsubishi Tanabe Pharma, MSD, Roche, Sanofi and UCB, Speakers bureau: Speaker fees from Actelion, Bayer, Boehringer Ingelheim, Medscape, Pfizer and Roche, Georg Schett Speakers bureau: AbbVie, BMS, Celgene, Janssen, Eli Lilly, Novartis, Roche and UCB, Jörg Distler Grant/research support from: Boehringer Ingelheim, Consultant of: Boehringer Ingelheim, Paid instructor for: Boehringer Ingelheim, Speakers bureau: Boehringer Ingelheim DOI: 10.1136/annrheumdis-2020-eular.4489

\section{SAT0288} CHARACTERIZATION OF ANTI-AMINOACYL TRNA
SYNTHETASE AUTOANTIBODIES IN PATIENTS WITH
IDIOPATHIC INFLAMMATORY MYOPATHIES

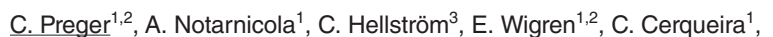
P. Nilsson ${ }^{3}$, I. E. Lundberg ${ }^{1}$, H. Persson ${ }^{4}$, S. Gräslund ${ }^{1,2}$, P. J. Jakobsson ${ }^{1,2}$. ${ }^{1}$ Karolinska Institutet, Karolinska University Hospital, Division of Rheumatology, Department of Medicine Solna, Stockholm, Sweden; ${ }^{2}$ Structural Genomics Consortium, Stockholm, Sweden; ${ }^{3}$ SciLifeLab, Royal Institute of Technology (KTH), Division of Affinity Proteomics, Department of Protein Science, Stockholm Sweden; ${ }^{4}$ SciLifeLab, Royal Institute of Technology (KTH), Division of Drug Discovery and Development, Department of Protein Science, Stockholm, Sweden

Background: Idiopathic inflammatory myopathies (IIM) are rare chronic inflammatory diseases associated with high mortality and morbidity [1]. One sub-group of IIM, anti-synthetase syndrome (ASS), is characterized by the presence of autoantibodies that target aminoacyl transfer(t) RNA synthetases (aaRS), together with specific clinical manifestations such as myositis, interstitial lung disease (ILD), arthritis, mechanic's hand, Raynaud's syndrome and fever [2]. The most common anti-aaRS autoantibody, anti-Jo1 targeting histidyl tRNA synthetase (HisRS), is present in up to $20-30 \%$ of patients with IIM, and up to $90 \%$ of patients with myositis and ILD [3, 4]. Besides Jo1, there are today seven other identified autoantigens within the aaRS family.

Objectives: A large part of patients with IIM, including individuals with clinical manifestations indicating ASS, test seronegative to all known myositis specific autoantibodies. However, these patients could potentially harbor autoantibodies 